\title{
Comentarios sobre el Atlas de Monterrey, coordinado por Gustavo Garza Villarreal
}

\section{Raúl Benítez Zenteno*}

Hace algunos años, me pidieron un trabajo sobre un conjunto de estudios presentados en un seminario sobre la ciudad de México, mismo que sirvió como presentación de la publicación. Con anticipación había realizado una revisión del libro Atlas de la Ciudad de México, también coordinado por don Gustavo Garza Villarreal, lo que me hace suponer que el gobierno municipal de Monterrey y después estatal de Nuevo León, sabía del producto posible al encargar este Atlas de Monterrey. Respecto al Atlas de la Ciudad de México me permití emitir algunas conclusiones:

En la reciente publicación, se mostró la evolución de la gran ciudad y de algunos de sus problemas, en buena medida consecuencia de su tamaño, que resulta, directamente del mantenimiento de la irracional decisión de seguir a toda costa, sobre todo de la población, concepciones en donde lo que se ha perdido es la visión desde dentro. Los estímulos a la actividad económica se han buscado fuera, hipotecando el patrimonio común. Se importan patrones ajenos de consumo y en la práctica se ha llegado a una situación moderna de tributo, transfiriendo recursos y capital hacia afuera y, lo que es más grave, limitando, de manera sustantiva, la investigación científica y tecnológica, con lo cual se reduce la posibilidad de acceso a alternativas de competencia, con independencia en la toma de decisiones, para participar con beneficio en el escenario del orden económico internacional.

Todas las estimaciones indican cifras que deben llevar a reorientaciones sustantivas de las pautas de desarrollo urbano de tan absurda situación, lo que implica, bien lo sabemos, transformaciones radicales en la manera de pensar el futuro de la Nación.

La ciudad de México constituye uno de los ejemplos más monstruosos de degradación del hombre y de la naturaleza. Se ha perdido la determinación de salvaguardar el patrimonio básico y se reducen las formas más elementales de solidaridad. Los programas de desarrollo de la ciudad responden más a estilos personales de las autoridades, lo que impide incluso continuidades mínimas, en las decisiones sobre el funcionamiento urbano, en cada cambio de administración y gobierno [...]

La ciudad de México constituye el gran ejemplo de problemas acumulados; de decisiones benéficas sólo para unos cuantos; de carencia de servicios en proporciones muy elevadas de la pobla-

\footnotetext{
* Profesor-investigador del Instituto de Investigaciones Sociales-UNAM.
} 
ción, de niveles de bienestar muy insatisfactorios, y tales conclusiones no son válidas para Monterrey, en donde se da un comportamiento institucionalizado diferente de los grupos económicos y políticos que mantienen su hegemonía y limitada participación de la sociedad civil.

A la vez existe una cierta pauta más universalmente difundida, de laborar dentro del ámbito regional capitalista, con una visión, desde aquí, de lo que requiere el país, lo que ha llevado a la consulta obligada de decisiones nacionales. Nuevo León constituye un ámbito reconocido e identificado en el conjunto nacional. No debe extrañar la afluencia de migrantes en sus épocas de mayor crecimiento poblacional, que coincidieron con la etapa de la transición demográfica de descenso de la mortalidad, bien identificada en los trabajos del capítulo 4 de Rodríguez Urrutia, Garza, Madrigal y Zúñiga, que nos aportan la información necesaria para ubicar a Monterrey en el contexto mundial de ciudades y en el nacional; saber del crecimiento poblacional por crecimiento natural o por crecimiento social, con un cierto sentido de proceso. No estoy diciendo que se llegó a la explicación de los procesos; estoy diciendo que se logró en cada caso una clara ubicación de las situaciones y sus cambios, lo que constituye la antesala de las explicaciones $\mathrm{y}$, en consecuencia, de lograr mayor claridad respecto a la construcción del futuro. Gustavo Garza lo dice mejor en su trabajo en el capítulo 9, sobre el escenario 1: Monterrey, metrópoli de crecimiento controlado: "Determinar con rigor su desarrollo futuro requiere de un profundo análisis de las estructuras socioeconómicas que lo han determinado en el pasado." (Y yo agregaría también a las políticas), y continúa: "Únicamente se intenta, por ende, hacer una reflexión general sobre su posible expansión económica y urbanística al año 2010 [...]" (p. 483). En este sentido, se da, con objetividad y cierta modestia, la indicación de un camino a seguir, que consiste a mi juicio en la creación de un espacio permanente de análisis científico social de carácter multidisciplinario, en donde, tomando en cuenta todo el escenario nacional y a los actores sociales y políticos regiomontanos, se construya la imagen de la ciudad del futuro que se desea para Monterrey. O sea, a partir del Atlas de Monterrey cuyas aportaciones son considerables, hay que ponerlas a prueba en la construcción del futuro, para que la urbe y su entorno regional, sean lo que sus pobladores busquen para sus descendencias, en un gran esfuerzo para no ser sólo una consecuencia de tendencias que confluyen en lo que ahora es Monterrey, y sí ser, por lo contrario, el resultado de una cierta racionalidad colectiva que tuvo la posibilidad de orientar su ciudad en términos de un gran colectivo concebido globalmente. 
Me parece que lo que algunos urbanistas han concebido como la ciudad global refiriéndose a Nueva York, Londres y Tokio, como centros de decisiones respecto al empleo de recursos financieros y la presencia de actividades super especializadas (cuaternarias y quinarias), dentro del carácter de la economía mundial actual, debe ser considerado como un escenario posible, que se vislumbra. Garza nos dice: "El crecimiento futuro de la ciudad podría no corresponder a esa vocación histórica." (p. 489). (Garza se refiere a la tradicional especialización industrial de Monterrey que ha sido, nos dice con justa razón, la ordenadora y generadora del espacio urbano actual). Y se plantea la creación de un nuevo polo de desarrollo como alternativa para garantizar un funcionamiento eficiente, lo que corresponde a la repetición de un ciclo que rememora el tránsito, que nos reseña el Atlas, de ciudad comercial a industrial de la primera mitad del siglo $\mathrm{xx}$.

Como bien puede colegirse, este excelente trabajo, resultado de un grupo amplio de expertos, despierta el requerimiento de ver el futuro desde un presente que se nos escapa por la rapidez de los cambios que tienen lugar, y que nos enfrenta exigiendo el abandono de la posición de espectador. De ahí la importancia de ser concretos, lo que me lleva a cumplir, con mayor cercanía, el encargo de referirme en especial a los capítulos cuatro, siete y nueve.

Don Fernando Antonio Rodríguez Urrutia nos ilustra al mostrarnos el escenario mundial y los problemas de las conceptualizaciones, previniéndonos de establecer similitudes entre ciudades de igual tamaño en entornos de desarrollo muy diferentes. Esta sola observación nos lleva a considerar los contextos de la urbanización en escala global. En una panorámica mundial, Monterrey aparece como ombligo del mundo según la nota explicativa, lo que a mí me parece un honor muy dudoso. Nos habla don Fernando Antonio de la importancia del medio ambiente. En este trabajo se insiste en el señalamiento del papel estratégico de Monterrey por razones geográficas, lo que debe, sin duda, ser tomado en cuenta en todos los análisis socioeconómicos.

Don Gustavo Garza en este capítulo nos muestra de nueva cuenta el fuerte hilo conductor de sus trabajos, y apunta el requerimiento de la descentralización, a lo que se hará mención después.

El amigo de muchos años, para no decirle el viejo amigo Romeo Madrigal Hinojosa, nos especifica los ritmos de la transición demográfica en Monterrey, y quiere dar a Monterrey la primacía en todo, hasta en la fecundidad más baja del país en 1989. Información más depurada nos dice que el Distrito Federal lleva el primer lugar. En mortalidad el estado de Nuevo León tiene la esperanza de vida 
más alta del país, tal y como lo señala Romeo. Para 1992, Nuevo León tuvo una esperanza de vida de 69.4 para hombres y 74.1 para mujeres, mientras que el Distrito Federal tuvo 69 y 73.5 respectivamente en una estimación de tablas de mortalidad cortada hacia los 85 años para establécer un horizonte de comparabilidad más certero (véase Demos, núm. 7, 1964). La pregunta para explicar una diferencia tan pequeña, con una mayor heterogeneidad y desigualdad en la ciudad de México, sería: ¿la atención de la salud es relativamente menor en Nuevo León?, o el nivel de bienestar es menor tal y como nos señalan las estadísticas de ingreso. Aquí vale la pena un señalamiento: dada la distancia entre la región centro y el norte en cuanto a ingreso monetario (40\% menor en promedio, véase Cortés y Rubalcava, 1994), la eficiencia del sistema de salud es mayor en Nuevo León. Si vemos para 1992 el rubro de causas de muerte, las enfermedades transmisibles materna y perinatal son muy parecidas entre las dos entidades; las no transmisibles también son muy parecidas; en donde el Distrito Federal tiene una mortalidad un poco menor es en el rubro de causas de muerte por lesiones y accidentes. Parecería que aquí aflora la diferencia.

Don Víctor Zúñiga nos expone el complejo panorama de la migración, en donde lo que sobresale es que no obstante la disminución del impacto de la migración de 1980 a 1990, todo señala un proceso nuevo en el que, nos dice el autor, el área metropolitana simultáneamente es receptora y expulsora de migrantes, y las ciudades medias del sistema de ciudades del noreste constituyen nuevas opciones tanto para migrantes de la región como de otras zonas del país. En otro trabajo de las ciudades medias en 1987 se evidencia (Corona y Tuirán et al., 1993) la disminución de la inmigración intraestatal en Monterrey; 75\% de los inmigrantes en 1987 viene de otros estados y, a diferencia del resto de las ciudades intermedias, se manifiesta un predominio de la inmigración masculina, con una gran brecha en la educación de inmigrantes y nativos, aunque la diferencia en este rubro es menor que la brecha que se da en el Área Metropolitana de la Ciudad de México. Uno de cada dos inmigrantes en Monterrey procede de los estados vecinos de San Luis Potosí (20\%), Coahuila (17\%) y Tamaulipas $(13 \%)$. Esto debe tenerse muy en cuenta dado que la población tiende a abandonar condiciones insatisfactorias por mejores expectativas.

Don Jesús Ramones Saldaña presenta el último trabajo de este cuarto capítulo, quien seguramente se congratuló de la existencia de encuestas de empleo urbano, ya que la información de los censos muestra, en este rubro de ocupación y actividad, las mayores 
deficiencias. Hay disminución del empleo y sin duda esto se ha agudizado en los últimos años. Paralelamente se destaca el aumento de la participación en actividades remuneradas de la mujer. De este trabajo me vi obligado a revisar el de doña Esthela Gutiérrez Garza, en el capítulo 3, para saber un poco más de lo que sucede en este aspecto. La manufactura en el Área Metropolitana de Monterrey (АмM) observa problemas de competitividad en su tránsito de restructuración productiva.

El balance de su desempeño en el comercio internacional ha sido muy heterogéneo, presentando en general una creciente dependencia de insumos y bienes de capital extranjeros que pueden significar un debilitamiento de su posición competitiva industrial.

Elevado déficit comercial en 1992. Nos expresa la autora que se

evidencia la enorme brecha de competitividad que existe en la gran mayoría de las ramas industriales del extranjero[...] En el contexto de las políticas de globalización, este problema es prácticamente general y manifiesta contradicciones microeconómicas y desajustes de las formas institucionales vigentes derivados de las políticas neoliberales de desarrollo (p. 147).

El empresario regiomontano sólo participó de 1988 a 1992 con $6 \%$ del total nacional en la inversión de maquinaria y equipo en el país, lo que es insuficiente, y se orientó más a la adquisición de la banca. En términos productivos se suscita una creciente dependencia de tecnologías extranjeras. La recuperación económica que se manifestó en Nuevo León de 1988 a 1991, pasó a ser recesiva en 1992 y 1993. Frente a este panorama se propone continuar la investigación ya que resulta grave la erosión de la competitividad de las empresas fabriles.

Me queda poco tiempo para los capítulos 7 y 9; seré breve. El séptimo capítulo constituye un detallado relatorio de la estructura y funciones del gobierno del estado y el gobierno municipal por Aldo Fasci Zuazua; la evolución de los órganos de gestión del área metropolitana se aborda por tres autores: Gustavo Garza, Celso Paniagua y Fernando Rodríguez Urrutia, en el que se preguntan por las alternativas futuras de gestión metropolitana y logran aclarar situaciones con base en diversos trabajos. Por ejemplo, el énfasis en la autonomía local no resulta muy adecuado en situaciones metropolitanas. Exponen cómo en Japón han creado gobiernos metropolitanos. Otra variante se da en Canadá. En Estados Unidos no hay gobiernos metropolitanos y hay en cambio instancias o gerencias 
de gestión urbana, y esto resulta en una compleja e intrincada relación intergubernamental. En el futuro del Amm hay que repensar muy bien este problema. Posiblemente pueda resolverse mediante participaciones democráticas ampliadas y representativas.

Don Edilberto Cervantes Galván nos introduce al sistema político y a las elecciones municipales de 1976 a 1991. La asistencia a las urnas es limitada y esto debe ser motivo de preocupación de los partidos y la ciudadanía.

Como contrapartida, el ciudadano se expresa en movimientos sociales con demandas específicas, nos dice doña María de los Ángeles Pozas Garza, lo que "responde a la incapacidad de las instituciones públicas para satisfacer las demandas", hecho que a la vez refleja una situación generalizada en el país que va en aumento. Sin dejar de ser expresión de la sociedad civil, en la década pasada se desarticularon los movimientos populares, mismos que "carecen de fuerza y capacidad para incidir políticamente". Todos los movimientos de la década de los setenta se diluyeron en los ochenta. Se ha llegado al establecimiento de una etapa de negociaciones con los distintos estratos sociales, incluidos los nuevos grupos del movimiento ecologista, a lo que, agrego yo, sólo quedan, frente a frente, los grupos de la iniciativa privada, empresariales y la banca, y el sector público. A mí, me habría gustado conocer un poco más de algunos problemas como, por ejemplo, las invasiones. Los estudios en la ciudad de México muestran con claridad el proceso de invasiones organizadas con capital de fraccionadores; se presiona y se logran autorizaciones. Posteriormente se compra a muy bajos precios a los invasores, se urbaniza y se vende con ganancias extraordinarias. Entre los beneficiados aparecen nombres de familias porfirianas y de nuevos políticos sobre todo del periodo alemanista.

Paso rápido al capítulo $\mathbf{9}$, considerando al $\mathbf{8}$, en donde se plantean dos grandes hipótesis sobre el futuro: ¿consolidación metropolitana o descentralización intraestatal?

De nueva cuenta, don Romeo Madrigal Hinojosa, nos plantea las perspectivas futuras con hipótesis claras. Surgen tres proyecciones: la histórica, la programática previa del Conapo y tres alternativas o escenarios de una tercera proyección. Por mi parte habría desechado la programática del Conapo publicada en 1985, por el hecho de que no contiene la información de 1990 y no corrigió adecuadamente la de 1980 , y además en el programa nacional de población de 1989-1994 se cambió la meta nacional al año 2000 de $1.0 \%$ a $1.5 \%$, lo que permitió que nuestro anterior presidente expresara en la última reunión oficial de población que se habían cumplido las metas. 
Romeo Madrigal nos presenta su proyección programática con tres escenarios, lo que lleva a confusiones dado que lo más probable es que las dependencias de gobierno podrán emplear cualquiera de ellas y tenderán a guiarse por la intermedia. Romeo lo resuelve: entre 3.25 y 3.5 millones de habitantes en el AMM, y sus tres escenarios quedan dentro de esta banda de confianza que no es muy amplia, sobre todo por el comportamiento de la migración. Me parece que debe haber un sola proyección programática a perseguir, que no es precisamente la más posible sino la más deseable, lo que nos obliga a relacionar lo socioeconómico con lo demográfico y plantear algunos problemas de ética social y ética pública. Me quedé con el interés de conocer la estructura por edad de la población, por grandes grupos de edad, por ejemplo, o grupos de edad muy significativos para la planeación, para asomarnos a otros problemas como el crecimiento de la población escolar y los requerimientos educativos; desde luego la población económicamente activa y sus transformaciones sectoriales posibles; el crecimiento de la población de la llamada tercera edad, que se acentúa, y hay que prever desde ahora su salud y su seguridad social. También hay que incursionar desde otras perspectivas; por ejemplo, dar orientaciones más específicas sobre los requerimientos de salud mediante el análisis de las causas de muerte y los años oue se dejan de vivir por enfermedades oque debieron superarse hace tiempo.

En los dos últimos temas del noveno capítulo se inquiere al futuro. Don Gustavo Garza hace un recorrido rápido de la historia reciente. La industria básica en el esquema de sustitución de importaciones imprimió una dinámica productiva que llevó a surtir el mercado local, el regional, el nacional y el internacional. Se indujo un elevado ritmo de crecimiento demográfico como resultado del nexo entre desarrollo industrial y urbano.

Con base en la proyección de su posible desarrollo económico, se elaboró el primer escenario demográfico y urbanístico del AMM, partiendo de un crecimiento económico moderado. Así, con un crecimiento del PIв de $3.5 \%$ se llega a una población de $3.3 \mathrm{mi}$ llones en el año 2000 y 4.2 millones en el año 2010 , o sea entre el escenario B programático y la proyección histórica (4.1 y 4.2 millones respectivamente).

Al escribir estas líneas y reconsiderar él texto, resuelvo que hay algo que no me gusta y es la hipótesis histórica como crecimiento constante de $2.55 \%$ para el АмM, basada sólo en la experiencia de 1980 a 1990, cuando se da una reducción marcada del crecimiento total (de $5.9 \%$ de 1960 a 1970 , a $2.51 \%$ de 1980 a 1990); lo anterior corresponde a un descenso sustantivo de creci- 
miento natural (de $3.4 \%$ a $2.0 \%$, muy parecido al nacional), y a un cambio radical del crecimiento social de $2.5 \%$ a $0.5 \%$ en las décadas consideradas. Y no me gusta porque no se hicieron explícitos estos componentes del crecimiento por separado y, por otra parte, si se mantiene el crecimiento económico, aunque moderado, también habrá expansión de la migración, dado que en el escenario regional y en el nacional es difícil esperar dicho crecimiento económico sostenido con el modelo neoliberal, sin desplazamiento de fuerza de trabajo, lo que lleva a una tendencia de mayor desigualdad económica y social, con una población que tiende a desplazarse sólo si se crean nuevas oportunidades en un entorno diferente. Es decir, si el crecimiento natural disminuye a 1.55 para el año 2000 , el crecimiento social se recuperará por lo menos a $1.0 \%$ de acuerdo con la hipótesis.

Me llama la atención y suscribo la propuesta de Garza sobre su lógica de desconcentración en la medida en que se obliga a incorporar más premisas a la planificación urbana. Y es aquí cuando me parece que surge con mayor claridad la propuesta de continuidad del esfuerzo de aunar más y más conocimiento sistemático y riguroso a la toma de decisiones, cada vez más abierta y técnicamente sustentada.

Me parece que hay que continuar por lo menos en cuatro áreas sustantivas: requerimientos educativos en número y sobre todo calidad de la enseñanza; fuerza de trabajo en razón de una traducción mínima de productividad y conocimiento, y conocimiento y cultura, dimensión humana. Conviene aquí el establecimiento de escenarios alternativos por la vía de consideraciones sobre el comportamiento de la actividad al nivel de dos dígitos de la clasificación internacional; un tercer gran tema es el de la salud y la seguridad social, alrededor del cual todos estamos de acuerdo en que constituye uno de los grandes sectores del bienestar. Conviene tratarlo, entre otros, por grandes grupos de edad y por causas de muerte como indicador directo del bienestar social. El cuarto gran tema es el del medio ambiente bajo la premisa de medio ambiente sustentable.

Por fin llegamos al último gran escenario de lo posible con la pregunta ¿consolidación metropolitana o megalopolización de Monterrey?, presentado por don Roberto García Ortega y Juan Ignacio Barragán, los que inician su trabajo con las consideraciones necesarias de la actual coyuntura nacional, sin precedentes. Ellos dicen que la premisa básica de la prospectiva es la de que el futuro no existe, y esto lo encuentro como parte de un esquema formalista en extremo simple. Yo creo que el futuro sí existe, y ha guiado a los pueblos en toda la historia de la humanidad. Es la 
base de todas las religiones. Todo lo que se postula está en razón del futuro que mueve montañas. En lo individual el futuro rige decisiones como las de la familia y, en condiciones sistémicas conservadoras, sostiene la hipótesis histórica. De hecho, lo primero que confronta la planificación es la hipótesis histórica: es la hipótesis que hay que modificar, que derrotar. Con la hipótesis de consolidación metropolitana la población llega a ser $\mathbf{5 . 3}$ millones en el AMM, parecida a la hipótesis histórica 10 años después. Tanto esta hipótesis como la de megalopolización tienen un gran peso hacia la reflexión en varios sentidos, y lo que expongo ahora tiene un carácter provocador. En primer lugar el crecimiento de Monterrey correspondió a la expansión mundial de posguerra, en buena medida para cubrir requerimientos de Estados Unidos y Europa, y este ciclo parece irrepetible sobre todo en cuanto al requerimiento de mano de obra en los contextos nacionales, de acuerdo con el modelo neoliberal. Una segunda reflexión se refiere a los planteamientos optimistas en los ámbitos gubernamentales respecto a "una economía que está en vías de estabilizarse". El argumento descansa en la disminución de los niveles de consumo para mantener los precios bajos y disminuir el ritmo de crecimiento inflacionario. A nivel nacional la IP señaló que las empresas no aguantarán a que los mercados financiero y cambiario se estabilicen. Señalaron que el Banco de México enfrenta el dilema de controlar la inflación o evitar la desaparición de la planta productiva para preservar la estabilidad sociopolítica. En ambas situaciones, si agregamos la restricción migratoria internacional, la población que se agrega a los que han perdido sus fuentes de empleo tenderá a permanecer, de ahí que habrá que insistir en soluciones demográficas y paliativos sociales. La IP en uno de sus análisis más críticos plantea que el programa crediticio internacional no evitará la recesión ni resolverá la postración de la planta fabril, el incremento del desempleo, el desplome del poder adquisitivo de la población y, mucho menos, el avance de la cartera vencida de empresas y particulares.

Como se ve, la declaratoria de los diversos sectores puede llevar al recrudecimiento de los antagonismos y enconos políticos, nos advierte un desplegado de muy reconocidos intelectuales (viernes 24 de marzo, 1995).

Todo lo anterior tiene que ver con la toma de decisiones si se quiere crecer y sobre todo crecer a $6 \%$ anual en el PIB, lo que resulta difícil de lograr. La respuesta necesaria obliga a pensar en otras alternativas, incluyendo la de metropolización y megalopolización al mismo tiempo; leídos los antecedentes, considero que ello se dará, lo que parece deseable, y nos lleva a convertir esta posibili- 
dad en hipótesis programable, es decir, buscada como conveniente para todos, con tendencia a no ampliar las desigualdades sociales de la metrópoli.

Me queda tan sólo una reflexión: en nuestras situaciones regionales se desestima la formación de recursos humanos hacia la investigación científica y tecnológica, tanto en la universidad pública como en la privada, y sobre todo no se da el apoyo sostenido y a mediano y largo plazos de un desarrollo institucional que haga posible mantener la investigación y la formación de nuevos recursos humanos. En ocasiones se tiende a pensar en términos muy especializados, es decir, en planeación regional, estatal o municipal, en lugar de expertos con una formación teórico-metodológica que le permita incursionar en cualquier nivel. Esfuerzos como el que se presenta ahora del Atlas de Monterrey, trabajo excelente, son muy escasos y esporádicos.

Hago votos por que este trabajo sea el inicio de una nueva época que enfrente el requerimiento de conocimiento para la toma de decisiones respecto al futuro de nuestras ciudades.

\section{Bibliografía}

Corona, Rodolfo y Rodolfo Tuirán et al. (1993), "Las ciudades medias y el fenómeno migratorio", informe preparado por El Colegio de la Frontera Norte para la Secretaría de Desarrollo Social (mimeo).

Cortés, Fernando y Rosa Ma. Rubalcava (1994), El ingreso de los hogares, México, Instituto Nacional de Estadística, Geografía e Informática/El Colegio de México/Instituto de Investigaciones Sociales-unam. 